\title{
Endoscopic retrograde cholangiopancreatography in infantile cholestasis
}

\author{
Mark L Wilkinson, Giorgina Mieli-Vergani, Colin Ball, Bernard Portmann, Alex P Mowat
}

\begin{abstract}
The difficulty of distinguishing surgically correctable causes of conjugated hyperbilirubinaemia in infants from other causes means that some infants may undergo laparotomy and intraoperative cholangiography unnecessarily, and others may be referred for surgery too late. In an attempt to improve the diagnostic accuracy in infants with conjugated hyperbilirubinaemia when standard methods produced equivocal results, we have been using prototype paediatric duodenoscopes (PJF 7.5 and XPJF 8.0; Olympus) to perform endoscopic retrograde cholangiopancreatography (ERCP). From 159 infants with conjugated hyperbilirubinaemia, 11 were referred for ERCP, which was performed in nine. In four in whom bile ducts were definitely visualised laparotomy was avoided. Operative cholangiography confirmed patent bile ducts in one in whom visualisation had been uncertain. Three of four in whom bile ducts were not seen had extrahepatic biliary atresia. Visible bile drainage in the fourth excluded atresia. No major complications ensued but there was radiological evidence of gall bladder perforation in one (common hepatic duct block) and overinflation with air was a problem until finer cannulae (Wilson-Cook) were introduced. In appropriately selected patients with conjugated hyperbilirubinaemia, ERCP with paediatric duodenoscopes in experienced hands may provide useful diagnostic information.
\end{abstract}

Conjugated hyperbilirubinaemia in infants may be due to intrahepatic disease or, less commonly, disorders of the extrahepatic bile ducts. It may occur in association with a wide range of metabolic, endocrine, infectious, or familial disorders. Particular difficulty may occur in distinguishing three relatively common disorders: idiopathic neonatal hepatitis, arteriohepatic dysplasia, and extrahepatic biliary atresia (EHBA). The first two conditions have a good prognosis with medical management, but the last requires urgent surgical treatment. Hepatoportoenterostomy by a skilled surgeon performed before 8 weeks of age produces a normal plasma bilirubin concentration in approximately $85 \%$ of infants, but with later surgery this is achieved in only one third. ${ }^{1}$ Eighty seven per cent of the children who have cleared the jaundice after surgery are alive with a good quality of life 15 years later. ${ }^{2}$ Without successful bile drainage these infants die of cirrhosis, usually by 2 years of age, ${ }^{3}$ unless liver transplan- tation is available. Such patients constitute the major paediatric indication for liver transplantation.

To date, a prelaparotomy diagnosis of EHBA is made by demonstrating complete cholestasis and by systematically excluding other disorders, particularly endocrine or genetic diseases such as $\alpha_{1}$ antitrypsin deficiency or cystic fibrosis, which may give appearances on a liver biopsy specimen suggestive of EHBA. Even if assessed by a histologist skilled in liver pathology of infancy there remains $10-15 \%$ of the cases in which either the changes in the specimen are equivocal or associated features such as congenital heart disease (for example, peripheral pulmonary stenosis) cause diagnostic uncertainty. In our experience, a correct diagnosis is obtainable in about $90 \%$ of the cases. ${ }^{4}$ For the remainder, laparotomy with intraoperative cholangiography should confirm or exclude EHBA, but in practice even an experienced surgeon may remove small but patent ducts in infants with severe cholestasis due to intrahepatic disease. To avoid unnecessary laparotomy, since November 1987 we have attempted to demonstrate patency of the extrahepatic bile ducts by endoscopic retrograde cholangiopancreatography (ERCP), using prototype neonatal duodenoscopes, in cases of conjugated hyperbilirubinaemia and complete cholestasis in whom the diagnosis was uncertain.

\section{Patients and methods}

Over the period November 1987 to January 1990,159 infants with conjugated hyperbilirubinaemia were admitted for diagnosis and management to the paediatric liver service of King's College Hospital.

Investigations included standard haematological and liver function tests, ultrasound examination of the liver and biliary system, screening for viral and bacterial infections, $\alpha_{1}$ antitrypsin phenotype, sweat test, methyl bromoiminodiacetic acid (methyl bromida) scan, liver biopsy, and bone marrow examination. In 104 infants the jaundice was considered due to intrahepatic disease and proved to be so on follow up. Forty four were considered to merit laparotomy and this decision was correct in all cases. In 11 infants with complete cholestasis (acholic stools, no excretion on methyl bromida scan) the clinical and pathological features were equivocal, the liver histology showing mixed hepatitic and biliary features, the latter falling short of a clear picture of biliary atresia. ERCP was performed in nine of these infants (six boys) at a median age of 13 weeks (range 7-42) and at a median weight $4 \cdot 45 \mathrm{~kg}$ (range $3 \cdot 7-6 \cdot 2$ ). For 
Clinical details and ERCP findings of the nine children investigated

\begin{tabular}{lllll}
\hline Sex & Age (weeks) & Weight $(\mathrm{kg})$ & $\begin{array}{l}\text { ERCP findings } \\
\text { (filling of pancreatic duct/common bile duct) }\end{array}$ & Final diagnosis \\
\hline F & 20 & $5 \cdot 6$ & Pancreatic duct only & EHBA \\
M & 8 & $4 \cdot 3$ & Pancreatic duct only & EHBA \\
F & 11 & $4 \cdot 5$ & Pancreatic duct only & EHBA \\
M & 42 & $6 \cdot 2$ & Both (common hepatic duct block) & ? \\
B & 13 & $3 \cdot 7$ & Both (hypoplastic biliary tree) & Arteriohepatic dysplasia \\
F & 19 & $4 \cdot 5$ & Pancreatic duct only (free bile drainage) & Arteriohepatic dysplasia \\
M & 22 & $3 \cdot 4$ & Both (normal) & Idiopathic neonatal hepatitis \\
M & 9 & $3 \cdot 7$ & Pancreatic duct only ? (equivocal filling) & Idiopathic neonatal hepatitis \\
\hline
\end{tabular}

EP =Endoscopic retrograde cholangiopancreatography

EHBA $=$ extrahepatic biliary atresia.

the other two infants either the operator (MLW) or the endoscope were unavailable.

The endoscopes used were specially designed Olympus OES duodenoscopes PJF $7 \cdot 5$ (in six patients) and XPJF 8.0 (in three patients) of 7.5 and $8.0 \mathrm{~mm}$ external diameter respectively. The biopsy channel of each of these endoscopes was of $2 \mathrm{~mm}$ calibre but the PJF 7.5 lacked a bridge/ elevator mechanism. Cannulae were of the tapered tip type. In one case cannulation could only be achieved by the use of a fine 'pre-cut' knife as a steerable cannula (Olympus). In the last patient a 3FG cannula was employed (Wilson-Cook). Non-ionic radiography contrast medium (Niopam 300) was used. The procedure was performed by a single endoscopist under general anaesthesia in eight infants and under sedation in one. Patients were intubated while in the supine position, sometimes with the help of a laryngoscope, and ERCP was thereafter performed in the standard manner. However, the narrow confines of the duodenum in infants compound the difficulties of the procedure. With the PJF $7 \cdot 5$, 'blind' cannulation was sometimes necessary due to the lack of a 'bridge' mechanism.

\section{Results}

Successful cannulation of the papilla was obtained in all nine infants (table). In three,

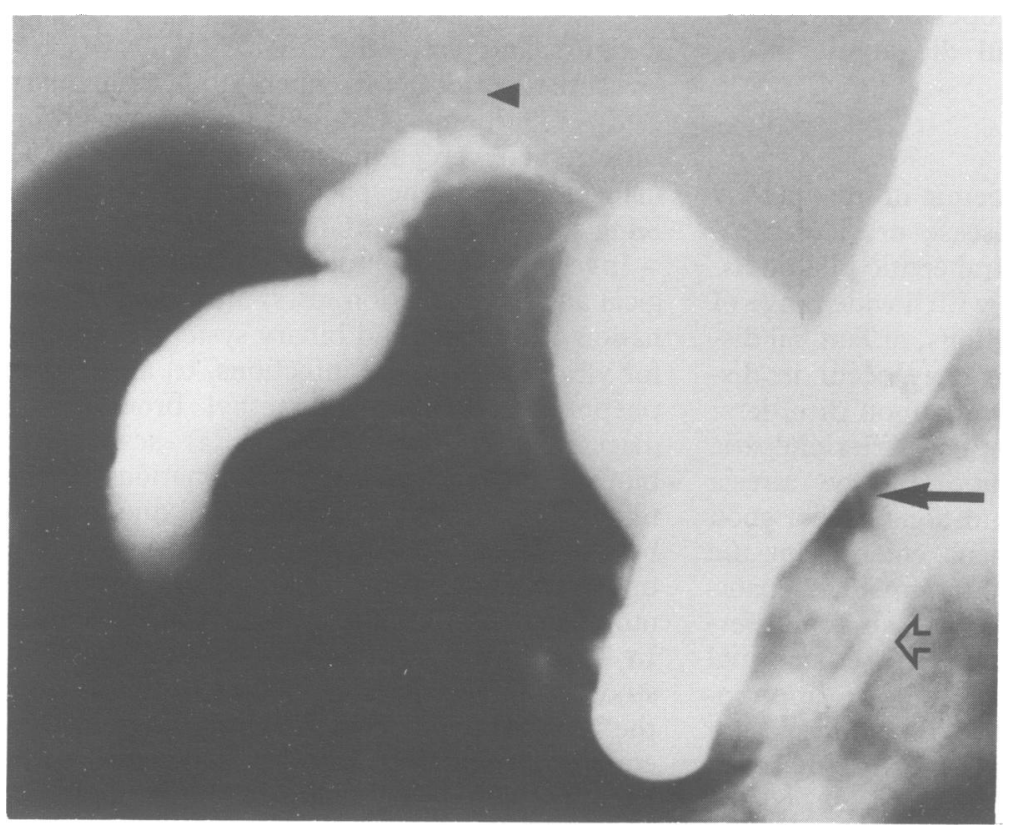

Endoscopic retrograde cholangiopancreatogram on a 19 week boy with comjugated hyperbilirubinaemia. Minute intrahepatic ducts (arrow head) and common bile duct (solid arrow), normal gall bladder, and pancreatic duct (hollow arrow) are shown. Eventual diagnosis was arteriohepatic dysplasia. only the pancreatic duct was demonstrated. In all of them EHBA was diagnosed at subsequent laparotomy and confirmed by histological examination of the excised tissue. Operative cholangiogram was not performed.

In one child there was simultaneous filling of the pancreatic duct and common bile duct, but no filling of the intrahepatic bile ducts and a common hepatic duct block was suspected. As the child had severe biliary type fibrosis and was already 42 weeks' old, it was decided not to proceed to a laparotomy in view of the possibility of a future liver transplant. The child was discharged with no definitive diagnosis. He was alive, having bled from oesophageal varices, at the age of 2.6 years and liver function tests showed features of compensated cirrhosis with a serum bilirubin concentration of $206 \mu \mathrm{mmol} / \mathrm{l}$, having been $230 \mu \mathrm{mo} / \mathrm{l}$ at the time of ERCP.

In one further infant pancreatic cannulation only was achieved, but bile was seen to be draining freely from the papilla before cannulation, and EHBA was thus excluded. The interval between the methyl bromida scan showing no secretion and the ERCP was 14 days. The jaundice subsequently cleared completely and, at the age of 1 year, hepatosplenomegaly persists but liver function tests are only mildly abnormal.

One child with severe giant cell hepatitis and moderate bile duct proliferation had a normal biliary tree at ERCP at 9 weeks of age. He progressed to chronic liver disease and died of liver failure at the age of 6 months. No aetiological factor was identified. His parents denied permission for a postmortem examination.

In one infant, aged 7 weeks, the liver biopsy specimen showed giant cell transformation with bile duct reduplication and mild portal tract oedema. ERCP showed a normal pancreatic duct, but no biliary filling could be seen on screening. After removal of the endoscope, a ductular structure in the region of the common bile duct and common hepatic duct, which had been hidden by the endoscope during the procedure, was seen. In view of the poor definition of the films, however, no firm conclusion could be made. The child underwent exploratory laparotomy with intraoperative cholangiogram, which showed patent intrahepatic and extrahepatic bile ducts. The child is improving and a provisional diagnosis of idiopathic neonatal hepatitis has been made.

Two infants had heart murmurs compatible with peripheral pulmonary artery stenosis and posterior embryotoxon (in one in association with butterfly vertebrae). Their liver biopsy 
specimens, though, showed cholangitic porta tract changes including oedema and bile duct proliferation. In one of them ERCP showed a normal pancreatic duct, cystic duct and gall bladder, but narrow common bile duct (maximum diameter of $0.48 \mathrm{~mm}$ ) and main hepatic duct branches $(<0.36 \mathrm{~mm})$ (figure). The films of the other patient were of poor quality because of spillage of contrast and inadequate injection, but they showed a normal calibre common bile duct and cystic duct, while the common hepatic duct was narrow, measuring only $0.3 \mathrm{~mm}$ in diameter. No intrahepatic ducts were seen, possibly due to poor filling. In both cases, a diagnosis of arteriohepatic dysplasia was made, supported by the subsequent development of hypercholesterolaemia and the typical facial appearance. At follow up both are well but with moderate to severe failure to thrive and abnormal liver function tests at the age of 31 and 32 months.

ERCP was carried out without serious complications in all nine cases. Despite minimal use of the air insufflator, however, abdominal distension was noted in all but the last patient, often making chest inflation by the anaesthetist difficult. The problem was due to the cannula completely filling the biopsy channel, thus preventing the use of the aspiration mechanism. Recently the use of finer cannulae, 3FG and 4FG (Wilson-Cook), has reduced this problem. In the patient with no duct filling beyond the common hepatic duct a small perforation of the gall bladder was seen radiologically but there were no clinical sequelae.

All infants recovered without problems from anaesthesia and were able to resume feeding the same day.

\section{Discussion}

Portoenterostomy is a life saving operation in patients with EHBA, particularly if performed before the age of 8 weeks. All efforts should be made to diagnose this condition as soon as possible. Exploratory laparotomy performed to exclude it in dubious cases should be avoided, however, because of possible deterioration of liver disease due to surgery and because it may compromise future hepatic transplantation. In the present study ERCP was able to diagnose or exclude accurately EHBA in eight of nine cases who otherwise would have required laparotomy.

The procedure was safe, although further modifications to the equipment may improve success and safety. The problem of abdominal distension during the procedure, which has caused a gastric perforation in a previous series, ${ }^{5}$ is reduced by the use of finer cannulae, which allow suction to take place during the procedure. ERCP was performed under general anaesthesia in most cases to avoid restlessness during the procedure. In only one child no definitive interpretation of the ERCP finding was possible, requiring further investigation by intraoperative cholangiogram.

Before 1987, ERCP was only occasionally described in neonates. ${ }^{6} 7$ In 1987 Guelrud et al reported a prospective study on unselected infants with conjugated hyperbilirubinaemia. ${ }^{8}$
Using a previous Olympus prototype they were successful in cannulating the biliary tree in 20 of 23 consecutive infants aged 2-21 weeks. They were able to diagnose bile duct obstruction in five with biliary atresia, normal biliary tree in 13 with neonatal hepatitis, and a choledochal cyst in one patient. In a further case with choledochal cyst ERCP did not visualise the common bile duct. In a study in 12 infants the following year, Heyman et al, also using a previous Olympus prototype, achieved cannulation only in four of 12 attempts. ${ }^{5}$ The poor success rate in that series is difficult to explain, but the authors were unable to find the papilla in three children. They suggested that papillary hypoplasia might be a consequence of neonatal hepatitis, though in the present series both cases with this diagnosis had normal papillae. Our results and those of Guelrud et al show that in infants it is usually impossible to cannulate the biliary and pancreatic ducts separately by appropriate manoeuvres of the cannula, unlike the situation in adults. In all four instances in our series and in 12 of 13 in that of Guelrud et al in which the bile duct was shown to be patent the pancreatic duct was also filled; no details of pancreatic opacification are given in the series of Heyman et al. On the other hand pancreatic duct visualisation and failure to opacify the biliary system in both our series and that of Guelrud et al was associated with a surgically correctable lesion of the biliary system in nine of 10 instances (EHBA in eight, choledochal cyst in one). In one of our infants, in whom the biliary system did not opacify at the same time as the pancreas, patency was assured as bile was seen to flow from the ampulla. In the two children with arterio hepatic dysplasia ERCP findings were distinctive, showing hypoplastic extrahepatic ducts and in one case very fine intrahepatic ducts.

There results suggest that if ERCP shows filling of the pancreatic duct without demonstration of the intrahepatic bile ducts laparotomy is essential. This must be performed by a surgeon with the skills and experience to diagnose and correct EHBA and other rarer biliary disorders and the confidence to leave hypoplastic but patent ducts in situ if the infant has intrahepatic disease. We conclude that ERCP using duodenoscopes designed for infants is a valuable addition to the management of selected children with conjugated hyperbilirubinaemia.

1 Mieli-Vergani G, Howard ER, Portmann B, Mowat AP. Late referral for biliary atresia-missed opportunities for effective surgery. Lancet 1989;i:421-3.

2 Ohkohchi N, Chiba T, Ohi R, Mori S. Long-term follow-up of patients with cholangitis after successful Kasai operation in biliary atresia: selection of recipients for liver transplantation. 7 Pediatr Gastroenterol Nutr 1989;9:416-20.

3 Ohi R, Hanamatsu M, Mochizuki I, Chiba T, Kasai M. Progress in the treatment of biliary atresia. World $\mathcal{f}$ Surg 1985;9:285-93.

4 Manolaki N, Larcher V, Mowat AP, Barrett JJ, Portmann B, Howard ER. The pre-laparotomy diagnosis of extrahepatic biliary atresia. Arch Dis Child 1983;58:591-4.

5 Heyman MB, Shapiro HA, Thaler MM. Endoscopic retrograde cholangiography in the diagnosis of biliary malformagrade cholangiography in the diagnosis of biliary ma.

6 Waye JD. Endoscopic retrograde cholangiopancreatography in the infant. Am $\mathcal{f}$ Gastroenterol 1976;65:461-3.

7 Urakami U, Seki H, Kishi S. Endoscopic retrograde cholan giopancreatography (ERCP) performed in children. Endosgiopancreatography

8 Guelrud M, Jaen D, Torres P, et al. Endoscopic cholangiopancreatography in the infant: evaluation of a new prototype pediatric duodenoscope. Gastrointest Endosc 1987;33:4-8. 